\title{
GABRIEL TOPOLOGIES ON DUBROVIN VALUATION RINGS
}

\author{
HIDETOSHI MARUBAYASHI \\ Department of Mathematics, Naruto University of Education, Naruto, Tokushima, Japan, 772-8502 \\ e-mail:marubaya@naruto-u.ac.jp \\ and YANG ZHANG \\ Department of Applied Mathematics, University of Western Ontario, London, ON, Canada, N6A 5B7 \\ e-mail: yzhang26@uwo.ca
}

(Received 3 February, 1999; accepted 26 February, 2001)

\begin{abstract}
All the Gabriel topologies on a Dubrovin valuation ring are classified in terms of its prime ideals. Furthermore, these Gabriel topologies are cogenerated by two kinds of indecomposable injective modules.
\end{abstract}

1991 Mathematics subject classification. 16D50, 16E60, 16H05.

Gabriel topologies have been investigated in the past 30 years by various authors with many applications to non-commutative ring theory. For commutative noetherian rings and commutative valuation rings, every Gabriel topology has a simple form in terms of the prime ideals (see [14, Chapter VII] and [1, Theorem 3.3]). However, in the noncommutative case, the situation is quite complex even if the ring is noetherian (see [14, Chapter VII]). In [8], a similar question is discussed for chain rings. In this paper, we classify in Theorem 5 all right Gabriel topologies on a Dubrovin valuation ring $R$ of a simple Artinian ring with finite dimension over its center by using prime ideals, which is a generalization of the commutative valuation ring case ([1, Theorem 3.3]). Our method is completely different from the commutative ring case and we use the henselization technique. As an application of the classification, we show that any right Gabriel topology on $R$ is naturally cogenerated by an indecomposable injective right $R$-module (Theorem 9 ).

Throughout this paper, we fix the following notation.

A ring will be an associative ring with unit element 1 . Let $\mathcal{C}$ be a regular Ore set of a ring $R$; that is, any element of $\mathcal{C}$ is regular and it is a right and left Ore set. We denote by $R_{\mathcal{C}}$ the quotient ring of $R$ with respect to $\mathcal{C}$. Let $M$ be a right $R$-module. An element $m$ in $M$ is called $\mathcal{C}$-torsion if $m c=0$ for some $c \in \mathcal{C}$. It is well known that the set $t_{\mathcal{C}}(M)$ of all $\mathcal{C}$-torsion elements of $M$ is an $R$-submodule of $M$. If $M=t_{\mathcal{C}}(M)$, then $M$ is called $\mathcal{C}$-torsion. On the other hand, if $t_{\mathcal{C}}(M)=0$, then $M$ is called $\mathcal{C}$-torsion-free. In the case in which $\mathcal{C}$ is the set of all regular elements of $R$, we simply say that a right $R$-module $M$ is torsion (resp. $M$ is torsion-free) if $M$ is $\mathcal{C}$-torsion (resp. $\mathcal{C}$ torsion-free).

Let $P$ be a prime ideal of $R$ and let $\mathcal{C}(P)=\{c \in R \mid c$ is regular mod $P\}$. If $\mathcal{C}(P)$ is a regular Ore set of $R$, then the localization of $R$ at $P$ is denoted by $R_{P}$. We denote the center of $R$ by $Z(R)$ and the Jacobson radical of $R$ by $J(R)$. As usual, $\operatorname{Spec}(R)$ is the set of all prime ideals of $R$. 
We recall that an order $R$ in a simple Artinian ring $Q$ is called a Dubrovin valuation ring if, for any $q \in Q \backslash R=\{q \in Q \mid q \notin R\}$, there exist $r, r_{1} \in R$ such that $q r, r_{1} q \in R \backslash J(R)$, and $R / J(R)$ is local; i.e., it is a simple Artinian ring.

Throughout this paper, $R$ will be a Dubrovin valuation ring of a simple Artinian ring $Q$ with finite dimension over its center $K$ and $V=Z(R)$, a valuation ring of $K$.

We shall frequently use the following properties of a Dubrovin valuation ring that have been proved by Dubrovin in [4] and [5].

(D1) The mapping $P \rightarrow \wp=P \cap V$, where $P \in \operatorname{Spec}(R)$, is a bijection between $\operatorname{Spec}(R)$ and $\operatorname{Spec}(V)$. The inverse mapping is given by $\wp \rightarrow P=J\left(R_{\wp}\right)$, where $\wp \in \operatorname{Spec}(V)([\mathbf{1 2}$, Theorem 7.8]).

(D2) For any $P \in \operatorname{Spec}(R), P^{n}=\wp R$, for some natural number $n$. If $P=P^{2}$, then $P=\wp R([\mathbf{1 2}$, Theorem 7.8 and Corollary 13.11]).

(D3) For any $P \in \operatorname{Spec}(R), \mathcal{C}(P)$ is a regular Ore set of $R, R_{P}=R_{\wp}$ and $P=J\left(R_{P}\right)([12$, Theorem 7.8]).

(D4) Let $P \in \operatorname{Spec}(R)$. Then $P$ is principal as a right (or left) $R_{\wp}$-module if and only if $P \neq P^{2}([\mathbf{1 2}$, Lemmas 6.9 and 13.1]).

(D5) $R$-ideals of $Q$ are linearly ordered by inclusions ([12, Proposition 6.4]).

Let $\Im$ be a right Gabriel topology on a ring $S$. Then there exists an injective right $S$-module $E$ satisfying $\mathfrak{I}=\left\{I\right.$ : right ideal of $\left.S \mid \operatorname{Hom}_{S}(S / I, E)=0\right\}$ by [14, Proposition 3.7 and Theorem 5.7, Chapter VI]. We say that $\Im$ is cogenerated by $E$ and that $E$ generates $\Im$. Let $I$ be a right ideal of a ring $S$ and $r \in S$. Then we use the following notation: $r^{-1} \cdot I=\{x \in S \mid r x \in I\}$. We refer the reader to [7] and [14, Chapter VI] for the definition and some elementary properties of right Gabriel topologies.

We begin with the following general properties of right Gabriel topologies. The first three lemmas are implicitly known. However we shall give the complete proofs, since we cannot find the proofs in any papers or any books.

Lemma 1. Let $A$ be an ideal of a ring $S$ and let $\Im$ be a right Gabriel topology on $S$. Set $\tilde{S}=S / A$. Then $\tilde{\Im}=\left\{J^{\prime} \mid J^{\prime}\right.$ is a right ideal of $\tilde{S}$ and $J^{\prime} \supseteq \tilde{I}$, for some $I \in \mathfrak{I}$, where $\tilde{I}=I+A / A\}$ is a right Gabriel topology on $\tilde{S}$.

Proof. Let $\tilde{r} \in \tilde{S}$ and $J^{\prime} \in \tilde{\Im}$. Then there exists $I \in \Im$ such that $J^{\prime} \supseteq \tilde{I}$. It is easy to check that $\tilde{r}^{-1} \cdot \tilde{I} \supseteq r^{-1} \cdot I$. Since $r^{-1} \cdot I \in \Im, \tilde{r}^{-1} \cdot J^{\prime} \in \tilde{\Im}$.

Next, let $\tilde{I} \in \underset{\tilde{I}}{\widetilde{I}}, I \in \mathfrak{I}$ and $\tilde{J}$ be a right ideal of $\tilde{S}$ such that $\tilde{a}^{-1} \cdot \tilde{J} \in \tilde{\Im}$ for any $a \in I$. Set $J=\varphi^{-1}(\tilde{J})$, where $\varphi: S \rightarrow S / A=\tilde{S}$ is the canonical homomorphism. Then it is clear that $\{r \in S \mid \tilde{a} \tilde{r} \in \tilde{J}\}=a^{-1} \cdot J \supseteq A$ for any $a \in I$. Hence $\tilde{a}^{-1} \cdot \tilde{J}=a^{-1} \cdot J$. Thus $a^{-1} \cdot J \in \mathfrak{I}$, and so $J \in \mathfrak{I}$. This implies that $\tilde{J} \in \tilde{\Im}$. Hence $\tilde{\Im}$ is a right Gabriel topology on $\tilde{S}$ by [14, Lemma 5.2, VI].

Let $\Im$ be a right Gabriel topology on a ring $S$ and let $M$ be a right $S$-module. An element $m \in M$ is said to be $\mathfrak{\Im}$-torsion if $m I=0$ for some $I \in \mathfrak{\Im}$. The set $t_{\Im}(M)$ of all $\Im$-torsion elements in $M$ is an $S$-submodule of $M$, and $t_{\Im}(M)$ is called the $\Im$-torsion submodule. If $t_{\Im}(M)=M$ or $t_{\Im}(M)=0$, then $M$ is called $\Im$-torsion or $\Im$-torsion-free, respectively. We say that $\mathfrak{\Im}$ is trivial if either all modules are $\mathfrak{\Im}$-torsion or $\mathfrak{\Im}$-torsionfree; that is, either $\mathfrak{\Im} \ni(0)$ or $\mathfrak{\Im}=\{S\}$. In the case of semiprime Goldie rings, we have the following result.

Lemma 2. Let $S$ be a semiprime Goldie ring with quotient ring $Q$, a semi-simple Artinian ring. Then any non-trivial right Gabriel topology on $S$ consists of essential right ideals of $S$. 
Proof. Let $\Im$ be a non-trivial right Gabriel topology on $S$ and assume that there exists $I \in \mathfrak{I}$ such that $I$ is not an essential right ideal of $S$. Then there is a uniform right ideal $U$ of $S$ such that $I \cap U=0$. By Zorn's Lemma, we can choose $J$ that is a maximal element in the set $\{H \mid H$ is a right ideal of $S, H \supseteq I$ and $H \cap U=0\}$. Then $J \in \Im$ and $J$ is a complement right ideal of $S$ in the sense of Goldie with $\operatorname{dim} S / J=\operatorname{dim} S-\operatorname{dim} J=1$. Here $\operatorname{dim} M$ is the uniform dimension of a module $M$. Since $S / J$ is torsion-free as a right $S$-module, we have a natural embedding: $S / J \hookrightarrow S / J \otimes Q$. Now let $\operatorname{dim} S=n$. Then $\Sigma^{n} \oplus S / J \hookrightarrow \Sigma^{n} \oplus S / J \otimes Q \approx Q$ as a right $S$-module and $Q$ is an essential extension of $\Sigma^{n} \oplus S / J$. Hence there exists $x \in \Sigma^{n} \oplus S / J$ such that $a_{n} n_{S}(x)=\{s \in S \mid x s=0\}=0$. This implies that $(0) \in \mathfrak{s}$, because $x$ is a $\Im$-torsion element, a contradiction. Hence $\mathfrak{I}$ consists of essential right ideals of $S$.

Lemma 3. Let $\mathfrak{I}$ be a right Gabriel topology on a ring $S$ and subset $\mathcal{C}$ of $Z(S)$ consisting of elements that are regular in $S$. Then $\mathfrak{\Im}_{\mathcal{C}}=\left\{I \mid I\right.$ is a right ideal of $S_{\mathcal{C}}$ such that $I \cap S \in \Im\}$ is a right Gabriel topology on $S_{\mathcal{C}}$, where $S_{\mathcal{C}}$ is the central localization of $S$ with respect to $\mathcal{C}$.

Proof. Let $I \in \Im_{\mathcal{C}}$ and $\alpha=s c^{-1} \in S_{\mathcal{C}}$, where $s \in S$ and $c \in \mathcal{C}$. Then $s^{-1} \cdot(I \cap S) \subseteq \alpha^{-1} \cdot I \cap S$ and $s^{-1} \cdot(I \cap S) \in \Im^{\text {. Hence }} \alpha^{-1} \cdot I \in \Im_{\mathcal{C}}$. Let $J$ be a right ideal of $S_{\mathcal{C}}$ such that $\alpha^{-1} \cdot J \in \mathfrak{I}_{\mathcal{C}}$ for any $\alpha \in I$. Then for any $a \in I_{0}=I \cap S$, we have $a^{-1} \cdot J \cap S \in$ I. Set $J_{0}=\Sigma_{a \in I_{0}} a\left(a^{-1} \cdot J \cap S\right)$, a right ideal. Then $a^{-1} \cdot J \cap S \subseteq a^{-1} \cdot J_{0}$ for any $a \in I_{0}$ implies that $J_{0} \in \mathfrak{I}$. Hence $J \in \mathfrak{I}_{\mathcal{C}}$ since $J_{0} \subseteq J$. Therefore $\mathfrak{I}_{\mathcal{C}}$ is a right Gabriel topology on $S_{\mathcal{C}}$ by $[\mathbf{1 4}$, Lemma 5.2, VI].

Let $P \in \operatorname{Spec}(R)$. Then we have the following two kinds of right Gabriel topologies on $R$ :

$\mathfrak{\Im}_{P}=\{I \mid I$ is a right ideal with $I \cap \mathcal{C}(P) \neq \emptyset\}$ and

$\bar{\Im}_{P}=\{I \mid I$ is a right ideal with $I \supseteq P\}$ if $P=P^{2}$.

Lemma 4. Let $R$ be a rank one Dubrovin valuation ring of $Q$ with $P=J(R)=P^{2}$, i.e., Spec $(R)=\{P,(0)\}$. Then any right Gabriel topology on $R$ is one of the following:

$\mathfrak{\Im}_{P}, \bar{\Im}_{P}, \mathfrak{\Im}_{(0)}$ and $\overline{\mathfrak{\Im}}_{(0)}$, where $\mathfrak{\Im}_{P}=\{R\}$ and $\bar{\Im}_{(0)}$ are trivial.

Proof. Let $\mathfrak{\Im}$ be any right Gabriel topology on $R$. If $P \notin \mathfrak{I}$, then $\mathfrak{\Im}$ does not contain any maximal right ideal and so $\mathfrak{s}=\mathfrak{\Im}_{P}=\{R\}$.

If $\Im$ contains a non-zero ideal $A$ different from $P$, then $0=\cap A^{n}$ by [12, Proposition 13.7 ], because $R$ is rank one. Let $B$ be any non-zero ideal of $R$. Then $B \supseteq A^{n}$ for some $n>0$ and so $\mathfrak{\Im} \supseteq \Im_{(0)}$ since $R$ is a prime PI ring. Hence either $\mathfrak{\Im}=\bar{\Im}_{(0)}$ or $\mathfrak{s}=\mathfrak{I}_{(0)}$ by Lemma 2 . Thus we may assume that $\mathfrak{s}$ does not contain non-zero ideals different from $P$ and that $P \in \mathfrak{I}$. It is clear that $\bar{\Im}_{P} \subseteq \mathfrak{\Im}$ and we shall prove that $\bar{\Im}_{P}=\mathfrak{s}$.

Assume that $\bar{\Im}_{P} \neq \Im$. Let $E$ be an injective right $R$-module that generates $\mathfrak{\Im}$ and let $V^{h}$ be the henselization of $V$. Then $V^{h} / V$ is a torsion-free $V$-module since $V^{h} \cap K=V$ and so $V^{h} / V$ is flat as a $V$-module (see [12, Lemma 11.6(1)]). It follows that $V^{h} / V=\lim _{\rightarrow} L_{i}$, the direct limit of $L_{i}$, where $L_{i}$ is a finitely generated free $V$-module. From the exact sequence $0 \rightarrow V \rightarrow V^{h} \rightarrow V^{h} / V \rightarrow 0$, we derive the exact sequence $0 \rightarrow E \rightarrow E \otimes V^{h} \rightarrow E \otimes V^{h} / V \rightarrow 0$. Since $E$ is injective, $E \otimes V^{h} \cong E \oplus\left(E \otimes V^{h} / V\right)$ as a right $R$-module. Note that $E \otimes V^{h} / V$ is a direct limit 
of a finite number of copies of $E$ since $V^{h} / V$ is torsion-free and $V$ is a valuation ring. Set $R^{h}=R \otimes V^{h}$. Then it is a Dubrovin valuation ring of $Q^{h}=Q \otimes K^{h}$ with $J\left(R^{h}\right)=$ $J(R) \otimes V^{h}$ by [12, Lemmas 4.4, 9.1, Theorems 9.8, 12.3], where $K^{h}=K \otimes V^{h}$. Note that $J\left(R^{h}\right)$ is also idempotent. Let $Q^{h}=M_{n}\left(D^{h}\right)$, an $n \times n$ matrix ring over $D^{h}$, where $D^{h}$ is a division ring. Since $V^{h}$ is a henselian valuation ring, there exists an invariant valuation ring $\Delta^{h}$ of $D^{h}$ with $Z\left(\Delta^{h}\right)=V^{h}$ (see [12, Corollary 8.3]). Since any onesided ideal of $\Delta^{h}$ is two-sided, it follows that there are four different kinds of right Gabriel topologies on $\Delta^{h}$. This is proved in exactly the same way as in the commutative case (see [1, Theorem 3.3]). By [12, Proposition 5.14], we may assume that $R^{h}=M_{n}\left(\Delta^{h}\right)$. Then there are four different kinds of right Gabriel topologies on $R^{h}$ by $[15, \S 2]$; that is, $\mathfrak{\Im}_{P^{h}}, \bar{\Im}_{P^{h}}, \mathfrak{\Im}_{\left(0^{h}\right)}$ and $\bar{\Im}_{\left(0^{h}\right)}$, where $P^{h}=P \otimes V^{h}$. Now, let $\Im^{h}$ be the right Gabriel topology on $R^{h}$ cogenerated by the injective hull of $E \otimes V^{h}$ as a right $R^{h}$-module. It is easily checked that $\Im^{h} \supseteq\left\{J \otimes V^{h} \mid J \in \Im \mathfrak{\Im}\right\}$ by [14, Proposition 5.5, VI]. Let $I$ be a right ideal of $R$ with $I \in \mathcal{I}$ and $I \notin \overline{\mathfrak{s}}_{P}$. Then we may assume that $I \subset P$ and so $I \otimes V^{h} \in \Im^{h}$. It follows from [12, Lemma 11.6] that $I \otimes V^{h} \cap R=I$, which implies that $I \otimes V^{h} \notin \overline{\mathfrak{\Im}_{P^{h}}}$. Thus $\mathfrak{\Im}^{h} \supseteq \Im_{\left(0^{h}\right)}$ follows. Hence for any non-zero ideal $A$ of $R$, we have $A \otimes V^{h} \in \Im^{h}$. But this implies by [14, Proposition 5.5, VI] that $x A \neq 0$ for any non-zero $x \in E$. Hence $A \in \mathfrak{s}$, a contradiction. Therefore $\mathfrak{s}=\bar{\Im}_{P}$.

Let $A$ be an ideal of $R$ with $P=\sqrt{A}$, the prime radical of $R$. It follows from [12, (13.1)] that $P=\sqrt{A}$ is a prime ideal of $R$. We say that $A$ is right primary if $x R y \subseteq A$, where $x, y \in R$, implies $x \in A$ or $y \in \sqrt{A}$. Similarly, a left primary ideal is defined, and an ideal $A$ is primary if it is right and left primary. Note that an ideal is right primary if and only if it is left primary. See [12, Corollary 13.4].

A prime ideal $P$ of $R$ is said to be branched if there exists a $P$-primary ideal different from $P$. If $P$ is the only $P$-primary ideal of $R$, then $P$ is said to be unbranched. Let $P$ be branched and let $A$ be $P$-primary with $A \neq P$. Then $P_{0}=\cap_{n=1}^{\infty} A^{n}$ is a prime ideal such that there is no prime ideal $P^{\prime}$ with $P \supset P^{\prime} \supset P_{0}$. We say that $P_{0}$ is the prime ideal next to $P$. If $P$ is unbranched, then $P=\cup\left\{P_{\lambda} \mid P_{\lambda} \in \operatorname{Spec}(R)\right.$ with $\left.P_{\lambda} \subset P\right\}$ (see [12, Theorem 13.10]).

Now we are in a position to prove the main theorem of this paper.

Theorem 5. Let $R$ be a Dubrovin valuation ring of a simple Artinian ring $Q$ with finite dimension over its center $K$ and let $\Im$ be a right Gabriel topology on $R$. Then there exists $P \in \operatorname{Spec}(R)$ such that either $\mathfrak{\Im}=\mathfrak{\Im}_{P}$ or $\mathfrak{\Im}=\bar{\Im}_{P}$ and $P=P^{2}$.

Proof. We may assume that $\Im$ is non-trivial; that is, there exists $I \in \Im$ with $I \neq R$ and $0 \notin \mathfrak{\Im}$. Then $\Im$ consists of essential right ideals of $R$ by Lemma 2 .

Consider the set $\mathcal{P}=\left\{P^{\prime} \in \operatorname{Spec}(R) \mid P^{\prime} \subseteq A\right.$ for any two-sided ideal $\left.A \in \Im\right\}$ and set $P=\cup_{P^{\prime} \in \mathcal{P}} P^{\prime}$. Then $P \in \operatorname{Spec}(R)$ by [12, Lemma 13.9]. It is easy to check the following facts:

(1) $P \subseteq A$ for any ideal $A \in \mathfrak{\Im}$.

(2) $P \supset P^{\prime}$ and $P^{\prime} \in \operatorname{Spec}(R)$ imply $P^{\prime} \in \mathcal{P}$.

We shall prove that either $\mathfrak{\Im}=\mathfrak{\Im}_{P}$ or $\mathfrak{\Im}=\bar{\Im}_{P}$ and $P=P^{2}$. The proof will be divided into several steps.

Step 1. We claim that if $B$ is an ideal of $R$ with $P \subset B$, then $B \in \Im$.

Define $P_{1}=\cap\left\{P^{\prime} \in \operatorname{Spec}(R) \mid P^{\prime} \supseteq B\right\}$. Since $P_{1} \supseteq B \supset P$, by the construction of $P$, there exists an ideal $A \in \mathfrak{\Im}$ such that $P_{1} \supset A$. For any $x \in P_{1} \backslash A=$ 
$\left\{x \in P_{1} \mid x \notin A\right\}$, set $I=R x R$. Then $I=r S=S r$ for some $r \in I$ by [12, Lemma 7.10], where $S=O_{r}(I)=\{q \in Q \mid I q \subseteq I\}$. Since $x \notin A, I \supset A$ and so $I \in \Im$ S. Since $I$ is principal as an $S$-module and $I \subseteq P_{1}$, by [12, Proposition 13.7], we have that $P_{2}=\cap_{n=1}^{\infty} I^{n}$ is a prime ideal and $P_{1} \supset P_{2}$. If $P_{2} \supseteq B$, then $P_{2} \supseteq P_{1}$ by the construction of $P_{1}$, a contradiction. Therefore $P_{2} \subset B$. Assume that $I^{n} \supseteq B$ for all $n$. Then $P_{2} \supseteq B$, a contradiction. Hence there exists $n$ such that $I^{n} \subseteq B$ and $I^{n} \in \mathfrak{\Im}$. Thus $B \in \mathfrak{\Im}$.

Step 2. We claim that $\mathfrak{\Im}_{P} \subseteq \mathfrak{\Im}$.

Set $\wp=P \cap V$. Then $R_{\wp}=R_{P}$ and for any $I \in \Im_{P}, I R_{\wp}=R_{\wp}$; i.e., there exists $c \in V \backslash \wp$ such that $c \in I$. Since $c \notin P, I \supseteq c R \supset P$. By Step 1, $c R \in \mathfrak{\Im}$ and so $I \in \mathfrak{\Im}$. Hence $\mathfrak{s}_{P} \subseteq \mathfrak{\Im}$.

Thus we have either $\mathfrak{\Im}_{P}=\mathfrak{\Im}$ or $\mathfrak{\Im}_{P} \subset \mathfrak{s}$.

Step 3. We claim that if $\mathfrak{\Im}_{P} \subset \mathfrak{\Im}$, then $\bar{\Im}_{P} \subseteq \mathfrak{\Im}$.

Let $E$ be the injective right $R$-module which cogenerates $\Im$. It follows from the assumption that there exists $I \in \mathfrak{s}$ such that $I R_{P} \subset R_{P}$, and so we may assume that $I$ satisfies the following:

$I=I R_{P} \cap R$ and $I R_{P}$ is a maximal right ideal of $R_{P}$.

Then we have an exact sequence: $0 \rightarrow R / I \rightarrow R_{P} / I R_{P}$. Assume that there exists a nonzero map $f: R_{P} / I R_{P} \rightarrow E$ and choose $0 \neq x=\left[r c^{-1}+I R_{P}\right] \in R_{P} / I R_{P}$ such that $f(x) \neq 0$, where $r \in R, c \in V \backslash \wp$. Since $c R \in \Im_{P} \subseteq \mathfrak{\Im}$ and $E$ is $\Im$-torsion-free by [14, Proposition 5.5, VI], we have that $0 \neq f(x) c=f(x c), x c \in R / I$. Hence $\left.f\right|_{R / I}$, the restriction map to $R / I$, is non-zero. This is a contradiction. Therefore, $\operatorname{Hom}\left(R_{P} / I R_{P}, E\right)=0$ and so $R_{P} / I R_{P}$ is $\Im$-torsion. Thus $R_{P} / P^{\prime}$ is also $\Im$-torsion, where $P^{\prime}=J\left(R_{P}\right)=P$. Since $R / P \subseteq R_{P} / P^{\prime}$, we have $P \in \mathfrak{\Im}$ and so $\bar{\Im}_{P} \subseteq \mathfrak{\Im}$.

Step 4. We claim that if $\bar{\Im}_{P} \subseteq \mathfrak{s}$, then $\bar{\Im}_{P}=\mathfrak{\Im}$.

We assume that $\bar{\Im}_{P} \subset \mathfrak{I}$ and consider the following three cases.

Case 1. Assume that $P \neq P^{2}$. Then $P=a R_{P}=R_{P} a$, for some $a \in R$, and so $P / P^{2} \cong R_{P} / P$. Note that we have proved that $R_{P} / P$ is $\Im$-torsion in Step 3. Thus $P^{2} \in \mathfrak{I}$, contradicting the choice of $P$. Hence it follows that $P=P^{2}$.

Case 2. $P=P^{2}$ and $P$ is branched.

Since $\overline{\mathfrak{s}}_{P} \subset \mathfrak{I}$, there exists $I \in \mathfrak{I}$ such that $I \notin \overline{\mathfrak{I}}_{P}$, and so $P \in \mathfrak{I}$ implies that $I \cap P \in \Im$. Thus we may assume that $I \subset P$. Furthermore, let $P_{0}$ be the prime ideal next to $P$ and set $\wp_{0}=P_{0} \cap D$.

If $I+P_{0}=P$, then $I_{\wp_{0}}+P_{\wp_{\wp_{0}}}=R_{\wp_{0}}$ and so $I_{\wp_{0}}=R_{\wp_{0}}$, by Nakayama's lemma. Thus there exists $c \in V \backslash \wp_{0}$ such that $c R \subseteq I$ and $c R \supset P_{0}$. Therefore $P \supset I \supset P_{0}$.

If $I+P_{0} \subset P$, then $P \supset I+P_{0} \supseteq P_{0}$ and $I+P_{0} \in \Im$. Hence, in both cases, we may assume that there exists $I \in \mathfrak{\Im}$ such that $P \supset I \supset P_{0}$.

Furthermore, if $I_{\wp}=P$, then for any $p \in P$ there exists $c \in V \backslash \wp$ with $p c \in I$. Since $c \notin P$, we have $c R \supset P$ and so $p P \subseteq p c R \subseteq I$. Thus $P=P^{2} \subseteq I$, a contradiction. Hence we may also assume that $I_{\wp} \subset P$. Then, by using Lemma 3, we may assume that $R=R_{\wp}$, i.e., $J(R)=P$.

Set $\tilde{R}=R / P_{0}$. Then $\tilde{R}$ is a rank one Dubrovin valuation ring with $\tilde{\widetilde{\Im}} \underset{\tilde{I}}{\supset} \bar{\Im}_{\tilde{P}}$, where $\tilde{P}=P / P_{0}$. It is easy to check that $\mathcal{C}(\tilde{P})=\widetilde{\mathcal{C}(P)}$. Hence $\tilde{\Im}_{P}=\Im_{\tilde{P}}$ and $\tilde{I} \subset \tilde{P}$ 
follows. Thus $\tilde{I} \in \tilde{\Im}$ but $\tilde{I} \notin \overline{\mathfrak{S}}_{\tilde{P}}$. Therefore, by Lemma 4 , either $\tilde{\widetilde{I}}=\mathfrak{\Im}_{(\tilde{0})}$ or $\tilde{\widetilde{\Im}}=\bar{\Im}_{(\tilde{0})} \supseteq \Im_{(\tilde{0}}$. This implies that for any ideal $A$ with $P_{0} \subset A \subset P, \tilde{A} \in \tilde{\Im}$ and so $A \in \mathfrak{I}$, a contradiction. Hence $\mathfrak{\Im}=\overline{\mathfrak{S}}_{P}$.

Case 3. $P$ is unbranched; that is, $P=\cup P_{\lambda}\left(P_{\lambda} \in \operatorname{Spec}(R)\right.$ with $\left.P_{\lambda} \subset P\right)$.

As in Case 2, there exists $I \in \Im$ with $I \subset P$. Assume that $I \in \Im_{P_{\lambda}}$ for all $\lambda$; that is, there exists $c_{\lambda} \in V \backslash \wp_{\lambda}$ such that $c_{\lambda} R \subseteq I$ for all $\lambda$. Set $A=\Sigma_{\lambda} c_{\lambda} R$, an ideal properly containing $P_{\lambda}$. Then we have $I \supseteq A \supseteq P$, a contradiction. Thus there is $P_{\lambda} \in \operatorname{Spec}(R)$ such that $P \supset P_{\lambda}$ and $I \notin \Im_{P_{\lambda}}$. If $I+P_{\lambda}=P$, then $I_{\wp_{\lambda}}+P_{\lambda_{\varphi_{\lambda}}}=P_{\wp_{\lambda}}=R_{\wp_{\lambda}}$, where $\wp_{\lambda}=P_{\lambda} \cap V$. This implies that $I_{\wp_{\lambda}}=R_{\wp_{\lambda}}$, a contradiction. Thus we may assume that $P \supset I \supseteq P_{\lambda}, I \in \Im$ and $\tilde{I}=I / P_{\lambda}$ is not an essential right ideal of $\tilde{R}=R / P_{\lambda}$. Since $\tilde{R}$ is a prime Goldie ring, we see from Lemma 2 that $\tilde{0} \in \tilde{\Im}$. This implies that $P_{\lambda} \in \Im$, a contradiction. Hence $\mathfrak{\Im}=\bar{\Im}_{P}$ follows.

Thus, we have proved that either $\mathfrak{I}=\mathfrak{\Im}_{P}$ or $\mathfrak{I}=\bar{\Im}_{P}$, for some $P \in \operatorname{Spec}(R)$. It remains to show that $P=P^{2}$ if $\Im=\bar{\Im}_{P}$. However, if $P \neq P^{2}$, then $P^{2} \in \Im$ as has been shown in Case 1. This is a contradiction. Therefore $P=P^{2}$.

Corollary 6. The set of all right Gabriel topologies on a Dubrovin valuation ring $R$ is linearly ordered by inclusion. In particular, if $P$ is an unbranched prime ideal, then $\bar{\Im}_{P}=\cap \Im_{P_{\lambda}}$, where $P_{\lambda} \in \operatorname{Spec}(R)$ with $P_{\lambda} \subset P$.

Proof. The first part follows from Theorem 5. Assume that $P$ is unbranched. Then it is clear that $\bar{\Im}_{P} \subseteq \cap \widetilde{\Im}_{P_{\lambda}}$. To prove the converse inclusion, let $I \in \Im_{P_{\lambda}}$ for any $P_{\lambda} \in \operatorname{Spec}(R)$ with $P_{\lambda} \subset P$. Then since $I_{\wp_{\lambda}}=R_{\wp_{\lambda}}$, there exists $c_{\lambda} \in V \backslash \wp_{\lambda}$ with $c_{\lambda} \in I$. Set $A=\sum c_{\lambda} R$. Then $A \subseteq I$ and $A \supset P_{\lambda}$, for all $P_{\lambda}$ with $P_{\lambda} \subset P$. Hence $A \supseteq P$ and so $A \in \bar{\Im}_{P}$. Therefore $I \in \bar{\Im}_{P}$.

For any right $R$-module $M$, we denote by $E_{R}(M)$ (or simply $E(M)$ ) the injective envelope of $M$. Let $E$ be an indecomposable injective right $R$-module. Then it is easy to see that $E$ is either torsion or torsion-free. If $E$ is torsion, then $E=E(R / I)$ for some meet-irreducible, essential right ideal $I$, and if $E$ is torsion-free, then $E$ is isomorphic to a minimal right ideal of $Q$.

The following lemma is due to Matlis [13] in the case of commutative domains.

Lemma 7. Let $I$ and $J$ be essential right ideals of $R$.

(1) Assume that $E(R / I)$ is indecomposable. Then $E(R / I) \cong E(R / J)$ if and only if $s^{-1} \cdot I=t^{-1} \cdot J$, for some $s \in R \backslash I$ and $t \in R \backslash J$.

(2) Assume that $I \subseteq J(R)$. Then

(a) $E(R / I)=E(Q / I)$

(b) $E(R / I) \cong E(R / c I)$, for any regular element $c \in R$.

Proof. (1) This is well known (see [10, Proposition 2.2]).

(2) (a) It suffices to prove that $Q / I$ is an essential extension of the right $R$-module $R / I$. Let $\bar{q}=[q+I]$ be any non-zero element of $Q / I$, where $q \in Q \backslash R$. Then there exists $r \in R$ with $q r \in R \backslash J(R)$. Since $I \subseteq J(R)$, it follows that $q r \notin I$ and so $\overline{0} \neq \bar{q} r \in R / I$.

(b) Since $c Q=Q$, for any regular element $c \in R$, the homomorphism $Q / I \rightarrow Q / c I$ given by $[q+I] \rightarrow[c q+c I]$ is an isomorphism as a right $R$-module. Hence we have $E(R / I)=E(Q / I) \cong E(Q / c I)=E(R / c I)$ by (a). 
We have shown in Theorem 5 that any right Gabriel topology $\mathfrak{I}$ on $R$ is either $\mathfrak{\Im}_{P}$ or $\bar{\Im}_{P}$ for some $P \in \operatorname{Spec}(R)$. Next we shall prove that $\mathfrak{s}$ is naturally cogenerated by the indecomposable injective right $R$-module $E$ with $P=\operatorname{Ass}_{R}(E)$, the associated prime ideal of $E$.

Let $M$ be a uniform right $R$-module. We define $\operatorname{Ass}_{R}(M)=\{r \in R \mid N r=0$, for some non-zero $R$-submodule $N$ of $M$ \}. It is clear that $\operatorname{Ass}_{R}(M)$ is an ideal of $R$.

Lemma 8. Let $M$ be a uniform right $R$-module. Then $\operatorname{Ass}_{R}(M)$ is a prime ideal of $R$.

Proof. Since $E(M)$ is uniform and $\operatorname{Ass}_{R}(M)=\operatorname{Ass}_{R}(E(M))$, we may assume that $M$ is an indecomposable injective right $R$-module. If $M$ is torsion-free, then $\operatorname{Ass}_{R}(M)=0$, a prime ideal, and so we may assume that $M$ is torsion. It follows from the proof of [6, Theorem 2.1] that $\wp=\operatorname{Ass}_{V}(M)=\left\{v \in V \mid a n_{M}(v) \neq 0\right\}$ is a prime ideal of $V$, because $a n n_{M}(v)=\{m \in M \mid m v=0\}$ is a right $R$-submodule of $M$. Since $M$ is $\mathcal{C}$-torsion-free, where $\mathcal{C}=V \backslash \wp$, it follows that $M$ is an injective right $R_{\wp}$ module (see [9, Corollary 9.16]). We shall prove that $P=A s s_{R}(M)$, where $P \in \operatorname{Spec}(R)$ with $\wp=P \cap V$. Since $\wp \subseteq \operatorname{Ass}_{R}(M)$, we have $\wp R \subseteq A s s_{R}(M)$. Assume that $\operatorname{Ass}_{R}(M)$ is not contained in $P$. Then $\operatorname{Ass}_{R}(M) \cap \mathcal{C}(P) \neq \varnothing$ and so $\operatorname{Ass}_{R}(M) \cap V \backslash \wp \neq \emptyset$, because $R_{P}=R_{\wp}$. Thus we have $\operatorname{Ass}_{V}(M) \supset \wp$, a contradication. Hence $\wp R \subseteq A \operatorname{Ass}_{R}(M) \subseteq P$ follows. We know that $P^{n}=\wp R$ for some $n>0$.

If $n=1$, then we have $P=A s s_{R}(M)$. If $n>1$, then $P \neq P^{2}$ and so $P=p R_{\wp}=R_{\wp} p$ for some $p \in P$, and there exists a non-zero $R$-submodule $N$ of $M$ such that $N p^{n}=0$. Hence we may assume that $N p^{m}=0$ and $N p^{m-1} \neq 0$ for some $m(1 \leq m \leq n)$. Then $0=N p^{m} R_{\wp}=N p^{m-1} R_{\wp} p$. This implies that $p \in \operatorname{Ass}_{R}(M)$ and so $P=p R_{\wp} \subseteq \operatorname{Ass}_{R}(M)$. Hence $P=\operatorname{Ass}_{R}(M)$ follows. $\square$

REMARK. Let $E$ be an indecomposable injective right $R$-module with $P=\operatorname{Ass}_{R}(E)$. Then $E$ is an indecomposable injective right $R_{P}$-module as it is seen in the proof of Lemma 8 , because $R_{P}=R_{\wp}$, where if $P=(0)$, then $R_{P}=Q$. Furthermore, we have the following two types.

(A) There exists a non-zero $x \in E$ such that $x P=0$.

(B) $x P \neq 0$ for any non-zero $x \in E$.

Theorem 9. Let $R$ be a Dubrovin valuation ring of $Q$ and let $P \in \operatorname{Spec}(R)$.

(1) $\mathfrak{\Im}_{P}$ is cogenerated by an indecomposable injective right $R$-module $E$ such that $P=A \operatorname{As}_{R}(E)$ and $E$ is of type (A).

(2) Assume that $P^{2}=P$. Then $\bar{\Im}_{P}$ is cogenerated by an indecomposable injective right $R$-module $E$ such that $P=\operatorname{Ass}_{R}(E)$ and $E$ is of type (B).

Proof. (1) $\mathfrak{\Im}_{P}$ is cogenerated by $E(R / P)=E_{R_{P}}\left(R_{P} / P\right)$ (see [11, Lemma 8] and [10, Corollary 3.10]). Let $I^{\prime}$ be a maximal right ideal of $R_{P}$ (if $P=(0)$, then $R_{P}=Q$ ) and let $I=I^{\prime} \cap R$. It is clear that $R_{P} / I^{\prime}$ is an essential extension of $R / I$ as a right $R$ module, $E_{R_{P}}\left(R_{P} / P\right)$ is a finite number of copies of $E\left(R_{P} / I^{\prime}\right)$ and $P=A \operatorname{ss}_{R}\left(E\left(R_{P} / I^{\prime}\right)\right)$. Hence $\mathfrak{\Im}_{P}$ is cogenerated by $E(R / I)$ since $E(R / I)=E_{R_{P}}\left(R_{P} / I^{\prime}\right)$, which is indecomposable. It is also clear that $E(R / I)$ is of type (A).

(2) Case $1.0 \neq P=P^{2}$ and $P$ is branched. 
Set $\wp=P \cap V$ and let $P_{0}$ be the prime ideal of $R$ next to $P$. For any fixed $\alpha \in \wp \backslash \wp_{0}$, where $\wp_{0}=P_{0} \cap V$, set $A=\alpha R_{\wp}$ and define $\operatorname{Ass}_{R}(E(R / A))=$ $\{r \in R \mid N r=0$ for some non-zero $R$-submodule $N$ of $E(R / A)\}$. Since $R / A$ is $\mathcal{C}$-torsion-free, where $\mathcal{C}=V \backslash \wp, E(R / A)$ is a right $R_{\wp}$-module by [9, Corollary 9.16]. To prove that $P=\operatorname{Ass}_{R}(E(R / A))$, let $r \in \operatorname{Ass}_{R}(E(R / A))$ and assume that $r \notin P$. Then $I r \subseteq A$ for some right ideal $I$ with $I \nsubseteq A$, and so $I \subseteq I_{\wp} R_{\wp}=I_{\wp} R_{\wp} r R_{\wp}=$ $(I R r R)_{\wp} \subseteq A_{\wp}=A$, a contradiction, because $J\left(R_{\wp}\right)=P$. Hence $P \supseteq \operatorname{Ass}_{R}(E(R / A))$.

Conversely, let $p \in P$ and set $B=R_{\wp} p R_{\wp}$. Then $B^{n} \subseteq A$ for some $n>0$ by [12, Lemma 13.8] and so $B \subseteq \operatorname{Ass}_{R}(E(R / A))$. Thus $P=\operatorname{Ass}_{R}(E(R / A))$ follows. Since $R / A$ is an $n$-chain ring for some $n>0$ by [4, Proposition 1 and Theorem 4, $\S 1$, it follows that $R / A$ has a finite Goldie dimension, that is $E(E / A)=E_{1} \oplus \cdots \oplus E_{k}$ for some indecomposable injective right $R$-module $E_{i}(1 \leq i \leq k)$. It follows that $A s_{R}(E(R / A))=A s s_{R} E_{i}$ for some $i$. To prove that $E=E_{i}$ is of type (B), assume that $r P \subseteq A$ for some non-zero $[r+A] \in R / A \cap E$. Set $C=R_{\wp} r R_{\wp}$. Then we have $P=\wp R_{\wp} \supset C \supset A$, because $P=J\left(R_{\wp}\right)$. $C P \subseteq A=\alpha R_{\wp}$ and $P^{2}=P$ imply that $\alpha^{-1} C \subseteq O_{l}(P)=R_{\wp}$ by [12, Lemma 6.8], where $O_{l}(P)=\{q \in Q \mid q P \subseteq P\}$. Thus $C \subseteq A=\alpha R_{\wp}$, a contradiction. Therefore $E$ is of type (B). Let $\Im$ be a right Gabriel topology on $R$ cogenerated by $E$. Since $E$ is of type (B) with $P=A s s_{R}(E)$, it follows by $[14$, Proposition $5.5 \mathrm{VI}]$ that $P \in \mathfrak{\Im}$ and so $\mathfrak{\Im} \supseteq \overline{\mathfrak{s}}_{P}$. Assume that $\mathfrak{\Im} \neq \overline{\mathfrak{s}}_{P}$. Then, by Theorem 5 and Corollary $6, \mathfrak{s} \supseteq \mathfrak{\Im}_{P_{0}}$. Set $E=E(R / I)$ for some meet-irreducible right ideal $I$ of $R$. Then for any $\beta \in \wp \backslash \wp_{0}$, there exists $s \in R$ such that $s \beta \subseteq I$ and $s \notin I$. Thus we may assume that $\beta \in I$, by Lemma 7 . Since $\beta R \supset P_{0}$, it follows that $\beta R \in \mathfrak{\Im}_{P_{0}} \subseteq \mathfrak{s}$ and $I \in \mathfrak{s}$ follows. Hence $\operatorname{Hom}_{R}(R / I, E)=0$, a contradiction. Therefore $\mathfrak{s}=\overline{\mathfrak{s}}_{P}$.

Case 2. $P$ is unbranched; that is, $P=\cup\left\{P_{\lambda} \mid P_{\lambda} \in \operatorname{Spec}(R)\right.$ with $\left.P_{\lambda} \subset P\right\}$.

Let $P_{\mu}$ be any branched prime ideal with $P_{\mu} \subset P$. Then, as in Case 1, set $A=\alpha R_{\wp}$ for any fixed $\alpha \in \wp_{\mu} \backslash\left(\wp_{\mu}\right)_{0}$, where $\wp_{\mu}=P_{\mu} \cap V$ and $\left(\wp_{\mu}\right)_{0}=$ $\left(P_{\mu}\right)_{0} \cap V$.

To prove that $P=\operatorname{Ass}_{R}(E(R / A))$, let $p$ be any element in $\wp$. Then we have $E(R / A) \cong E(R / p A)$ by Lemma 7. Thus we may assume that $R / p A$ is embedded in $E(R / A)$. Now $[\alpha+p A]$ is non-zero with $[\alpha+p A] p=\overline{0}$ and thus $\wp \subseteq \operatorname{Ass}_{R}(E(R / A))$. Let $x=p r$ be any element in $P=\wp R$, where $p \in \wp$ and $r \in R$. Then there exists a right ideal $I$ of $R$ such that $I p \subseteq A$ and $I$ is not contained in $A$. Thus $I x \subseteq A$ and so $x \in \operatorname{Ass}_{R}(E(R / A))$. Hence $P \subseteq A s_{R}(E(R / A))$ follows. The converse inclusion follows as in Case 1. Therefore $P=\operatorname{Ass}_{R}(E(R / A))$. Now we can find an indecomposable injective right $R$-module $E$ of type (B) with $P=A s s_{R}(E)$ in exactly the same way as in Case 1 . Let $\Im$ be a right Gabriel topology on $R$ cogenerated by $E$. Then we have $\mathfrak{\Im} \supseteq \bar{\Im}_{P}$ as in Case 1 . Assume that $\mathfrak{\Im} \neq \bar{\Im}_{P}$. Then $\mathfrak{\Im} \supseteq \mathfrak{\Im}_{P_{\tau}}$ for some $P_{\tau} \in \operatorname{Spec}(R)$ with $P_{\tau} \subset P$, by Theorem 5 and Corollary 6. Since $P$ is unbranched, there exists a branched prime ideal $P_{\lambda}$ with $P \supset P_{\lambda} \supset P_{\tau}$. Note that $\left(P_{\lambda}\right)_{0} \supseteq P_{\tau}$. As in Case 1, there exists a meet-irreducible right ideal $I_{\lambda}$ and $\alpha_{\lambda} \in I_{\lambda} \cap \wp_{\lambda} \backslash\left(\wp_{\lambda}\right)_{0}$ such that $E=E\left(R / I_{\lambda}\right)$, where $\wp_{\lambda}=P_{\lambda} \cap V$ and $\left(\wp_{\lambda}\right)_{0}=\left(P_{\lambda}\right)_{0} \cap V$. Since $I_{\lambda} \supseteq \alpha_{\lambda} R \supset\left(P_{\lambda}\right)_{0}$, we have $\alpha_{\lambda} R \in \mathfrak{\Im}_{P_{\lambda 0}} \subseteq \mathfrak{s}$ and so $I_{\lambda} \in \mathfrak{s}$, a contradiction. Hence $\mathfrak{s}=\overline{\mathfrak{s}}_{P}$.

Finally if $P=0$, then it is clear that $\bar{\Im}_{(0)}$ is cogenerated by $E=\{0\}$, the zero $R$ module.

We shall give a more detailed structure of an indecomposable injective right $R$-module $E$ with $P=A s_{R}(E)$ in the case in which $R$ is Prüfer in a forthcoming paper. 


\section{REFERENCES}

1. W. Brandal and E. Barbut, Localizations of torsion theories, Pacific J. Math. 107(1) (1983), 27-37.

2. H. H. Brungs and J. Gräter, Valuation rings in finite-dimensional division algebras, J. Algebra 120 (1989), 90-99.

3. H. H. Brungs and J. Gr̈ater, Extensions of valuation rings in central simple algebras, Trans. Amer. Math. Soc. 317 (1990), 287-302.

4. N. I. Dubrovin, Noncommutative valuation rings, Trans. Moscow Math. Soc. 45 (1984), 273-287.

5. N. I. Dubrovin, Noncommutative valuation rings in simple finite-dimensional algebras over a field, Math. USSR. Sbornik 51(2) (1985), 493-505.

6. A. Facchini, Generalized Dedekind domains and their injective modules, J. Pure and Applied Algebra 94 (1994), 159-173.

7. Jonathan S. Golan, Topologies on a ring: an overview (Longman Scientific and Technical, Harlow, 1987).

8. Jonathan S. Golan; Ana M.de Viola-Prioli and Jorge E. Viola-Prioli, Ducompact filters and prime kernel functors, Comm. Algebra 22(12) (1994), 4637-4651.

9. K. R. Goodearl and R. B. Warfield, An introduction to noncommutative noetherian rings (Cambridge University Press, Cambridge, 1989).

10. J. Lambek and G. Michler, The torsion theory at a prime ideal of a right Noetherian ring, J. Algebra 25 (1973), 364-389.

11. H. Marubayashi, A Characterization of bounded Krull prime rings, Osaka J. Math. 15 (1978), 13-20.

12. H. Marubayashi, H. Miyamoto and A. Ueda, Non-commutative valuation rings and semi-hereditary orders, K-Monographs in Math. No. 3 (Kluwer Academic Publishers, 1997).

13. E. Matlis, Injective modules over Prüfer rings, Nagoya Math. J. 15 (1959), 57-69.

14. B. Stenstrom, Rings of quotients (Springer Verlag, 1975).

15. D. R. Turnidge, Torsion theories and rings of quotients of Morita equivalent rings, Pacific J. Math. 37(1) (1971), 225-234. 